\title{
Angio-Seal Vascular Closure Related Acute Limb Ischemia: A Case Report
}

\author{
Maya Srinivasan ${ }^{1}$, Pramod Theetha Kariyanna ${ }^{1}$, Jeremy Smith ${ }^{1}$, Sushruth Das $^{2}$, Amog Jayarangaiah ${ }^{3}$, \\ Sudhanva Hegde ${ }^{1}$, Jessica L. Perez Perez ${ }^{1}$, Isabel M. McFarlane ${ }^{1, *}$ \\ ${ }^{1}$ Division of Cardiovascular Diseases and Department of Internal Medicine, State University of New York, \\ Downstate Medical Center, Brooklyn, NY 11203, U.S.A. \\ ${ }^{2}$ Base PU College, Rajajinagar, Bangalore, India- 560010. \\ ${ }^{3}$ Trinity School of Medicine, Ratho Mill, Ribishi, St. Vincent and Grenadines \\ *Corresponding author: isabel.mcfarlane@downstate.edu
}

Received November 03, 2019; Revised December 09, 2019; Accepted December 25, 2019

\begin{abstract}
Vascular Closure Devices (VCD) are routinely used in cardiac catheterization and other endovascular procedures in order to achieve immediate post-procedural hemostasis and sealing of the femoral artery puncture site. Unlike manual compression, VCD encompass a broad range of devices, with varying mechanisms, that offer the advantage of achieving rapid hemostasis, increased patient comfort and mobility, decreased reliance on hospital staff resources, and facilitate earlier hospital discharge. Complications of VCD have been well-described and include embolization, arterial occlusion, infection, or vascular obstruction. Here, we describe a case in which the Angio-Seal device was utilized during an elective cardiac catheterization resulted in acute lower extremity ischemia.
\end{abstract}

Keywords: vascular closure devices, percutaneous coronary intervention, angio-seal device, embolization, vascular obstruction

Cite This Article: Maya Srinivasan, Pramod Theetha Kariyanna, Jeremy Smith, Sushruth Das, Amog Jayarangaiah, Sudhanva Hegde, Jessica L. Perez Perez, and Isabel M. McFarlane, "Angio-Seal Vascular Closure Related Acute Limb Ischemia: A Case Report.” American Journal of Medical Case Reports, vol. 8, no. 2 (2020): 49-52. doi: 10.12691/ajmcr-8-2-3.

\section{Introduction}

Percutaneous coronary angiography and intervention have become routine practice, essential to the diagnosis and management of coronary artery disease. Worldwide, seven million percutaneous procedures are performed annually. [1] Although deemed minimally invasive, percutaneous procedures involve puncture of an arterial site and accordingly carry risk of access-site complications including hematoma, pseudoaneurysm, arterial dissection, and arteriovenous fistula. Post-procedural percutaneous coronary intervention access site bleeding complications were associated with a 71 percent increase in peri-procedural mortality. [2] Thus, sufficient hemostasis at the arterial puncture site is of paramount importance to prevent procedure related morbidity and mortality. Traditionally, manual compression (MC) at the arterial site was utilized and involved a minimum of 30 minutes of compression followed by eight hours of bed rest. To improve recovery time, reduce hospital resource utilization, improve patient comfort, as well as facilitate earlier discharge, [1,3] vascular closure devices (VCD) were introduced in the 1990s and include a variety of devices implanted into the vessel wall to close the arterial access site. VCD are categorized by the mechanism in which they generate a seal and include (1) collagen-based technology
(2) suture-mediated technology, and (3) staple or clip-based technology. The Angio-Seal is a type of collagen VCD consisting of an extra-arterial collagen layer, an intra-arterial anchor comprised of polyglycolic and polylactic acids, and a bioabsorbable suture connecting the two layers. Through a sandwich technique, the Angio-Seal compresses the arterial access site and induces hemostasis via the collagenous sponge. [1]

\section{Case Report}

A 58-year-old female with a past medical history of atrial fibrillation refractory to two previous ablations not on anticoagulation therapy due to upper gastrointestinal bleeding, severe aortic stenosis, breast cancer with bilateral mastectomy, obesity, cirrhosis, esophageal varices with history of bleeding and end stage renal disease on hemodialysis presented to the Emergency Department for progressive dyspnea and decreased exercise tolerance over the course of several months. Given her history of aortic stenosis, transthoracic echocardiography was performed to evaluate severity of aortic stenosis as contributor to her progressive symptoms. Transthoracic echocardiography revealed mild to moderate right ventricular systolic dysfunction with normal left ventricular systolic function and small left ventricular volume, as well as mild mitral stenosis, severe 
aortic stenosis. In light of the echocardiogram findings, patient underwent an elective right and left cardiac catheterization via the right femoral artery to further define coronary anatomy and measure right heart and pulmonary artery pressures. After completion of the procedure, Angio-Seal was deployed to close the femoral arterial access site and manual hand pressure was placed over the access site for several minutes. Within minutes thereafter, the patient reported 10/10 throbbing pain of the right leg distal to the knee, associated with paresthesia to the lower limb. Physical examination revealed edematous right leg and foot and absent right dorsalis pedis and right popliteal pulses that were neither palpable nor identifiable via doppler ultrasound. Interventional Radiology and vascular surgery were urgently consulted given concern for acute lower limb ischemia. Right lower extremity angiogram was performed immediately, with images as below in Figure 1. Angiography demonstrated complete occlusion of the common femoral artery with the Angio-Seal device in the sub-intimal plane of common femoral artery (CFA) dissecting the calcific atheromatous plaque from the CFA and resulting in CFA thrombosis. Vascular surgery performed right groin exploration with right femoral artery cutdown, extraction of the Angio-Seal device, atherectomy, endarterectomy with patch angioplasty. Repeat right lower extremity angiography demonstrated restoration of blood flow to the right leg and foot. On physical exam however, dorsalis pedis pulses remained nonpalpable, but were detectable with doppler studies. Tissue plasminogen activator (tPA) and nitroglycerin were infused into the distal popliteal artery in efforts to maximize distal perfusion, and within 24 hours, pain had completely resolved, dorsalis pedis and popliteal pulses were palpable on physical exam, and no sensory or motor deficits were sustained. Patient was subsequently discharged without further event.

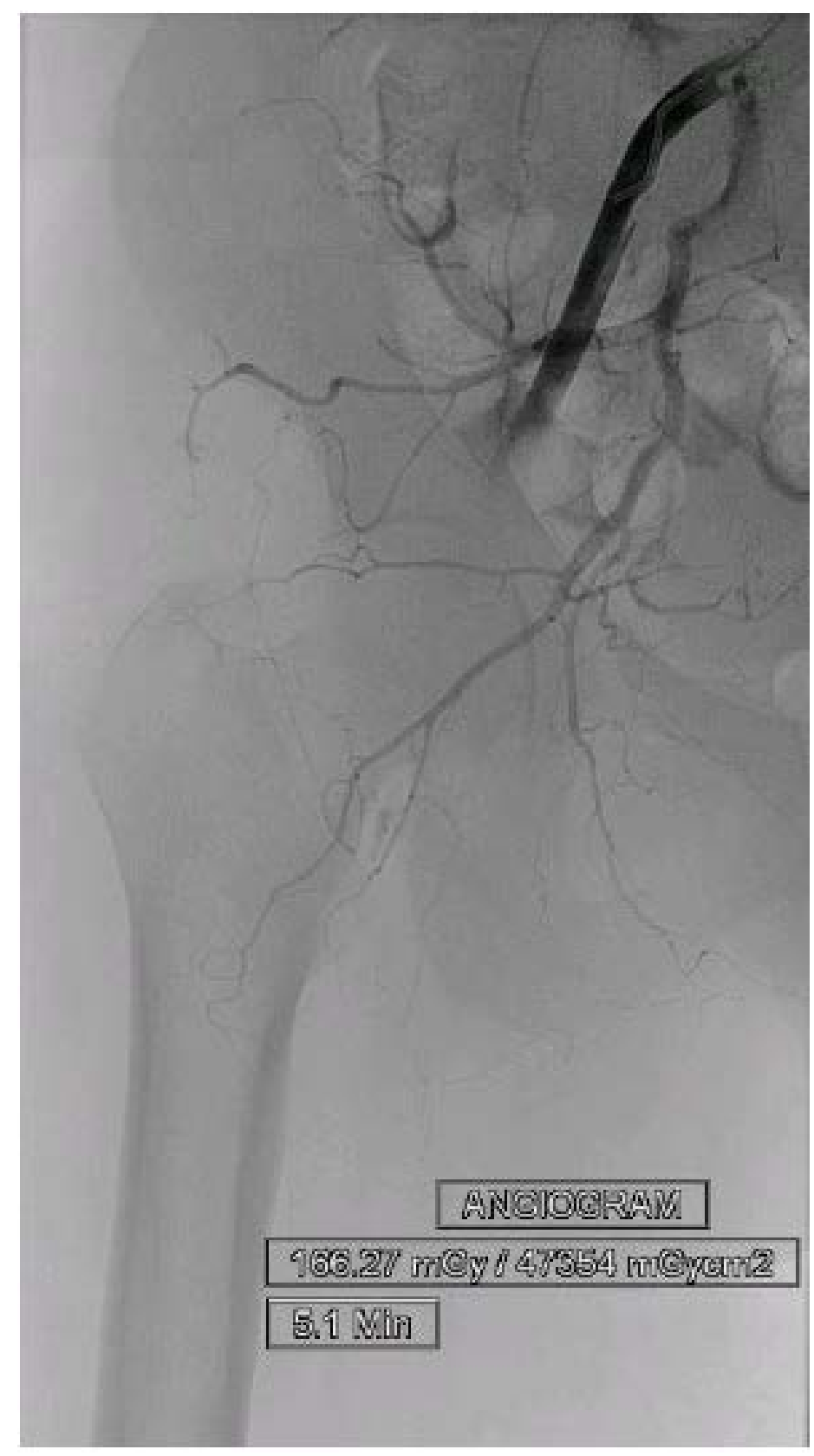

Figure 1. Angio-Seal device in the sub-intimal plane of CFA dissecting the calcific atheromatous plaque from the CFA; CFA thrombosis in the popliteal artery 


\section{Discussion}

VCD are used to achieve hemostasis in femoral catheterization. Multiple meta-analyses have demonstrated a similar risk profile of access-site related complications between VCD and the traditional gold standard of manual compression during diagnostic cardiac catheterization. $[4,5,6]$ VCD has maintained its prominence due to its additional advantages of decreased time to hemostasis, earlier time to ambulation, and earlier hospital discharges. Through these measures, VCD are associated with decreased resource utilization and translate to lower hospital costs. $[7,8,9]$

Vascular complications during cardiac catheterizations vary in severity and have decreased over time but the potential for complications is still nonetheless present. [10] VCD complications may include infection, occlusion of the artery, acute limb ischemia, as well as bleeding associated risk including arteriovenous fistula, hematoma, and pseudoaneurysm formation. Though estimates vary based on both the procedure whether diagnostic or interventional, as well as the specific device used, VCD failure resulting in occlusion of the artery occurs in $0.1-2.9 \%$ of cases 1 , with an estimated $0.06 \%$ causing complete obstruction resulting in acute limb threatening ischemia. [11-16] The Angio-Seal device offers one of the safer options with lower complication rates with comparison to other devices. [4]

While there are no clear contraindications to using the Angio-Seal device, there are recommendations about when they can safely be used. In non-urgent situations it is suggested that femoral arteriography can be done to measure the size of the artery and the integrity of the lumen. Some consider a vessel diameter less than five millimeters or luminal distortion within five millimeters of the puncture site to be a contraindication to Angio-Seal use. [17] With diagnostic and therapeutic catheterization procedures being done at more than seven million each year in the United States alone, patients such as ours with poor vasculature must have pre-procedural femoral arteriography to avoid undue morbidity and mortality. [18]

Preferential use of radial artery access instead of femoral arterial access may help reduce access-related complications such as bleeding. Studies have shown that using the radial artery as the arterial puncture site has fewer complications when compared to the femoral artery. $[19,20]$ With regard to the effectiveness and safety of manual hand compression versus VCD, there is strong evidence that the latter facilitates earlier discharges, however, without consistent evidence of improved outcomes with regards to complications.

\section{Conclusion}

VCD utilization during percutaneous procedures have become routine practice which has helped to improve patient discomfort and to limit unnecessary extended hospital stays. Often, cardiac catheterizations are performed in time-limited settings, in which pre-procedural arteriography is sacrificed. However, our presented case highlights the potential life-threatening consequences of VCD use without pre-procedural arteriography. Overall, trans-radial access is one approach with less risk for access-site related complications and should be preferentially employed, if the radial anatomy is permissive and the patient is not at risk for total radial artery occlusion. Alternatively, when the femoral access site is used, routine use of preprocedural arteriography must be incorporated in all cases in order to identify unfavorable vasculature and relative contraindications to minimize preventable complications.

\section{Acknowledgements}

This work is supported, in part, by the efforts of Dr. Moro O. Salifu M.D., M.P.H., M.B.A., M.A.C.P., Professor and Chairman of Medicine through NIH Grant number S21MD012474.

\section{References}

[1] Noori VJ, Eldrup-Jørgensen J. A systematic review of vascular closure devices for femoral artery puncture sites. Journal of Vascular Surgery. 2018; 68(3): 887-899.

[2] Kwok CS, Khan MA, Rao SV, et al. Access and non-access site bleeding after percutaneous coronary intervention and risk of subsequent mortality and major adverse cardiovascular events: systematic review and meta-analysis. Circ Cardiovasc Interv. 2015; 8(4).

[3] Robertson L, Andras A, Colgan F, et al. Vascular closure devices for femoral arterial puncture site haemostasis. Cochrane Database of Systematic Reviews 2016, Issue 3. Art. No.: CD009541.

[4] Nikolsky E, Mehran R, Halkin A, et al. Vascular complications associated with arteriotomy closure devices in patients undergoing percutaneous coronary procedures: a meta-analysis. J Am Coll Cardiol. 2004;44(6): 1200.

[5] Resnic F, Arora N, Matheny M, et al. A cost-minimization analysis of the angio-seal vascular closure device following percutaneous coronary intervention. Am J Cardiol. 2007; 99(6): 766-770.

[6] Schulz-Schüpke S, Helde S, Gewalt S, et al. Comparison of Vascular Closure Devices vs Manual Compression After Femoral Artery Puncture: The ISAR-CLOSURE Randomized Clinical Trial. JAMA. 2014; 312(19): 1981-1987.

[7] Marso S, Amin A, House J, et al. Association between use of bleeding avoidance strategies and risk of periprocedural bleeding among patients undergoing percutaneous coronary intervention. JAMA, 2010; 303(21).

[8] Khaldi A, Waldau B, Skowlund C, et al. Delayed Complication from a Percutaneous Vascular Closure Device Following a Neuro-Interventional Procedure. Interv Neuroradiol. 2011; 17(4): 495-500.

[9] Cox T, Blair L, Huntington C, et al. Systematic Review of Randomized Controlled Trials Comparing Manual Compression to Vascular Closure Devices for Diagnostic and Therapeutic Arterial Procedures. Surg Technol Int. 2015; 27: 32-44.

[10] Applegate, R., Sacrinty, M., Kutcher, M. et al. Trends in vascular complications after diagnostic cardiac catheterization and percutaneous coronary intervention via the femoral artery, 1998 to 2007. JACC. Cardiovascular Interventions, 1(3), 317-326.

[11] Vidi VD, Matheny ME, Govindarajulu US, et al. Vascular closure device failure in contemporary practice. JACC Cardiovasc Interv. 2012; 5(8): 837-844.

[12] Theodos G, Raymond C, Becker MC, et al. Arteriotomy closure device safety after percutaneous coronary intervention in the direct thrombin inhibitor era: a comparative study. Catheter Cardiovasc Interv. 2013; 81(2): 294-300.

[13] Biancari F, D’Andrea V, Di Marco C, et al. Meta-analysis of randomized trials on the efficacy of vascular closure devices after diagnostic angiography and angioplasty. Am Heart J. 2010; 159(4): 518-531. 
[14] Koreny M, Riedmüller E, Nikfardjam M, et al. Arterial puncture closing devices compared with standard manual compression after cardiac catheterization: systematic review and meta-analysis. JAMA. 2004 Jan 21; 291(3): 350-7.

[15] Bangalore S, Arora N, and Resnic F. Vascular Closure Device Failure: Frequency and Implications. A Propensity-Matched Analysis. Published October 27th 2009. Circulation: Cardiovascular Interventions. 2009; 2: 549-556.

[16] Wille J, Vos JA, Overtoom TT, et al. Acute leg ischemia: the dark side of a percutaneous femoral artery closure device. Ann Vasc Surg. 2006; 20(2): 278-281.

[17] Abando A, Hood D, Weaver F, et al. The use of the Angioseal device for femoral artery closure. J Vasc Surg. 2004; 40(2): 287-290.
[18] Kalapatapu VR, Ali AT, Masroor F, et al. Techniques for managing complications of arterial closure devices. Vasc Endovascular Surg. 2006; 40(5): 399-408.

[19] Romagnoli E, Biondi-Zoccai G, Sciahbasi A, et al. Radial versus femoral randomized investigation in ST-segment elevation acute coronary syndrome: the RIFLE-STEACS (Radial Versus Femoral Randomized Investigation in ST-Elevation Acute Coronary Syndrome) study. J Am Coll Cardiol. 2012; 60(24): 2481-2489.

[20] Jolly SS, Yusuf S, Cairns J, et al. Radial versus femoral access for coronary angiography and intervention in patients with acute coronary syndromes (RIVAL): a randomised, parallel group, multicentre trial. Lancet. 2011; 377(9775): 1409-1420.

(C) The Author(s) 2020. This article is an open access article distributed under the terms and conditions of the Creative Commons Attribution (CC BY) license (http://creativecommons.org/licenses/by/4.0/). 